\title{
ANÁLISE DESCRITIVA DAS VARIÁVEIS EQUILÍBRIO E FLEXIBILIDADE EM PARTICIPANTES DE UM PROGRAMA DE ORIENTAÇÃO E REEDUCAÇÃO POSTURAL EM GRUPO
}

Letícia Endsfeldz Teixeira, Jessica Jessica Ribeiro, Carolina Orsi, Giulia Iracelis Passarini da Silva, Débora Fernandes dos Santos, Tamara dos Santos Gouveia, Taíse Mendes Biral, Célia Aparecida Stellutti Pachioni, Dalva Minonroze Albuquerque Ferreira

Curso de Fisioterapia da Universidade Estadual Paulista Júlio de Mesquita Filho - Faculdade de Ciências e Tecnologia FCT/UNESP. E-mail: letícia_13_893@hotmail.com

\section{RESUMO}

O equilíbrio postural permite ao indivíduo manter o sistema músculo esquelético em posição estática e dinâmica, possibilitando uma postura adequada para o funcionamento eficaz das atividades cotidianas. A flexibilidade pode ser caracterizada pela amplitude articular máxima sem promover risco de lesões. O objetivo foi avaliar o equilíbrio e a flexibilidade de participantes do programa de orientação e reequilíbrio postural em grupo da Faculdade de Ciências e Tecnologia da UNESP - Campus de Presidente Prudente. O estudo transversal analisou 10 sujeitos (56 \pm 19 anos) que realizam atividades de alongamento, fortalecimento, equilíbrio, coordenação e conscientização corporal. Para a análise foi utilizado o teste do Banco de Wells e o Timed Up And Go. Os resultados demonstram que em relação à mobilidade funcional $100 \%$ dos participantes apresentam baixo risco de quedas e $70 \%$ boa flexibilidade. 0 programa beneficia seus participantes em relação à mobilidade funcional, à flexibilidade e ao equilíbrio dinâmico.

Palavras-chave: Alongamento; Flexibilidade; Equilíbrio; Orientação postural; Mobilidade funcional.

\section{INTRODUÇÃO}

Orientação postural diz respeito ao posicionamento relativo dos segmentos corporais, estando esses relacionados entre si e com o meio ambiente. A integração de várias estruturas como o sistema motor (força muscular, tônus muscular, reflexos tônico de postura); são essenciais para o funcionamento adequado do nosso corpo; dentre eles o equilíbrio (CARNAVAL, 2002).

Equilíbrio postural é o estado no qual todas as forças atuantes sobre o corpo estão equilibradas e o corpo tende a permanecer na posição e orientação desejada (equilíbrio estático), podendo se movimentar de uma forma controlada (equilíbrio dinâmico) (MANTOVANI et al, 2011).

Uma maneira de avaliar e identificar os déficits de equilíbrio é o teste Timed Up and Go (TUG) (PODSIADLO, RICHARDSON, 1991), sendo esse uma medida composta que envolve potência, velocidade, agilidade e equilíbrio dinâmico e objetiva avaliar a mobilidade funcional em atividades cotidianas (SHUMWAY, BRAUER, WOOLLACOTT, 2000).

Flexibilidade e o alongamento são qualidades essenciais para um bom desempenho físico e para a realização de atividades da vida diária. Uma boa flexibilidade é alcançada através da mobilidade e elasticidade adequada dos tecidos moles que circundam a articulação e pode ser 
garantida através do alongamento. Os alongamentos permitem a execução de movimentos com amplitudes articulares dentro de suas necessidades específicas, além de permitir a obtenção de arcos articulares mais amplos (BADARO, SILVA, BECHE, 2007; ALMEIDA, JABUR, 2007).

A flexibilidade muscular é avaliada através de movimentos que promovam o alongamento dos músculos. Uma das formas de avaliar essa variável é através do Banco de Wells, também conhecido como teste de sentar e alcançar, sendo usado na avaliação física para medir a amplitude do alongamento da cadeia mestra posterior (POLACHINI, 2005).

Existem vários métodos propostos, tanto para o tratamento postural, quanto para a melhora flexibilidade e equilíbrio; porém são métodos que referem ao tratamento individualizado. Embora pouco utilizado, o tratamento postural em grupo também é válido, e acredita-se na sua eficácia para melhoria das disfunções (HASHIMOTO et al, 2009). A atividade física em grupo, além de favorecer melhor aptidão física e socialização, pode vir a facilitar a adesão e motivação ao tratamento (MELLO, LUFT, MEYER, 2004).

O projeto de extensão intitulado "Programa de orientação e reequilíbrio postural em grupo (PORPOG)" realiza atividades que envolvem alongamento, fortalecimento, equilíbrio, coordenação e conscientização corporal, além de orientar quanto aos maus hábitos, prevenir posturas inadequadas durante as atividades laborais, promover a conscientização da postura e favorecer o relacionamento social em grupo (HASHIMOTO et al, 2009).

Diante do exposto acima, o objetivo deste estudo foi avaliar a flexibilidade e o equilíbrio de participantes do programa de orientação e reequilíbrio postural em grupo desenvolvido na Faculdade de Ciências e Tecnologia da UNESP - Campus de Presidente Prudente.

\section{METODOLOGIA}

Trata-se de um estudo transversal com 10 participantes, de ambos os gêneros que frequentam regularmente o PORPOG. Os participantes realizaram 36 sessões, durante 18 semanas, com frequência de duas vezes por semana e duração de 60 minutos cada uma, no ano de 2013. A cada sujeito foi entregue um termo de consentimento/esclarecimento possibilitando sua participação nesse estudo. O PORPOG é um projeto de extensão e este estudo científico foi aprovado pelo Comitê de Ética no Processo n $194 / 2007$.

Para mensurar a flexibilidade foi utilizado o teste linear do Banco de Wells ou teste de Sentar e Alcançar, sendo solicitada a flexão anterior da coluna vertebral com os membros inferiores em extensão, com a cabeça entre os braços também estendidos, mantendo-se estático 
a partir da posição de máximo alcance do movimento (FARIA, BARROS, 1998). Os resultados foram expressos em uma escala de distância em centímetros, sendo feitas três tentativas e considerada a de maior valor.

Para avaliação do equilíbrio dinâmico foi utilizado o teste Timed Up And Go (TUG), em que o indivíduo levanta-se de uma cadeira padronizada com apoio, porém sem auxílio dos braços, caminha 3 metros e retorna até o ponto de partida, sentando novamente. Para iniciar o teste, o mesmo recebeu a instrução "vai" e o tempo foi cronometrado a partir da voz de comando até o momento em que o mesmo voltou a sentar e o dorso da coluna foi apoiado no encosto da cadeira novamente. A partir disso, a mobilidade funcional é quantificada em segundos, determinando o risco de quedas (PODSIADLO, RICHARDSON, 1991).

A propensão a quedas foi avaliada através do tempo gasto para realizar o teste, sendo assim, menos de 10 segundos designando baixo risco de quedas; 10 a 20 segundos, médio risco de quedas e acima de 20 segundos, alto risco de quedas (PODSIADLO, RICHARDSON, 1991).

\section{RESULTADOS}

Foram analisados 10 sujeitos, destes, oito do gênero feminino (80\%) e dois do gênero masculino (20\%), com idade média e desvio padrão de $56 \pm 19$ anos, respectivamente.

$\mathrm{Na}$ avaliação da mobilidade funcional verificou que a média do tempo de execução dos participantes foi de $6,19 \mathrm{~s} \pm 1$, 2 . Já a flexibilidade avaliada pelo teste do Banco de Wells, obteve o valor médio de $29,15 \mathrm{~cm} \pm 11,7$ (Tabela 1 ).

Tabela 1. Valores da média e desvio-padrão da flexibilidade e equlibrio

\begin{tabular}{lcc}
\hline & Média & Desvio-padrão \\
\hline $\begin{array}{l}\text { Flexibilidade - Banco de } \\
\begin{array}{l}\text { Wells (cm) } \\
\text { Equilibrio - TUG (seg) }\end{array}\end{array}$ & 29,15 & 11,7 \\
\hline
\end{tabular}

Os resultados demostram que em relação à mobilidade funcional $100 \%$ dos participantes apresentam baixo risco de quedas. Com relação à classificação da flexibilidade, observou-se, na tabela 2, que a maioria dos participantes encontram-se entre excelente e média flexibilidade. 
Tabela 2. Porcentagem e classificação da flexibilidade dos participantes do programa de orientação e reequílibrio em grupo.

\begin{tabular}{lcl}
\hline & $\mathrm{N}^{\circ}$ de participantes & $\%$ \\
\hline Excelente & 4 & 40 \\
Média & 3 & 30 \\
Abaixo da média & 1 & 10 \\
Fraco & 2 & 20 \\
\hline
\end{tabular}

\section{DISCUSSÃO}

A postura mantem-se em constante adaptação e sua orientação está relacionada à estabilidade entre o indivíduo e o meio externo, envolvendo informações sensoriais e reações (BANKOFF et al, 2006). Na literatura, poucos estudos foram realizados envolvendo a temática do trabalho postural em grupo. Segundo o estudo de Martins et al (2010), a participação em grupo de postura contribuiu para melhora significativa na qualidade de vida e na capacidade funcional dos indivíduos.

Neste estudo, os resultados demonstraram que a maioria (70\%) dos participantes apresentou boa flexibilidade e esses dados corroboram com a classificação de Ribeiro et al. (2010), que mensurou o nível de flexibilidade de homens e mulheres de diferentes faixas etárias através do teste do Banco de Wells.

Segundo Bankoff et al (2006), uma postura correta é indispensável para um bom equilíbrio, mas uma postura não correta não implica obrigatoriamente num distúrbio do equilíbrio. Essa informação vem de encontro com a pesquisa realizada já que, de acordo com os resultados do teste TUG, todos os participantes do programa apresentaram um bom equilíbrio e baixo risco de quedas, apesar dos desvios relacionados à má postura.

Figueiredo, Lima, Guerra (2007) constatou que indivíduos adultos independentes e sem alterações no equilíbrio realizam o teste em 10 segundos ou menos; e os que são dependentes de alguma forma realizam o teste com mais de 10 segundos. Como constatado, este estudo obteve resultados positivos em relação à baixa propensão a quedas.

Nascimento, Vareschi, Alfieri (2008) relataram que um dos fatores relacionados à propensão de quedas é a questão da mobilidade funcional e o deslocamento no ambiente, sendo essas funções importantes para que os indivíduos realizem suas atividades de vida diária de maneira independente. Para amenizar este risco devem ser tomadas algumas medidas tais como: incentivo a prática regular de exercício físico e mudança no estilo de vida. 
Conforme Bertolla et al (2007), durante o ciclo da vida, o declínio dos níveis de flexibilidade vai gradativamente dificultando a realização de diferentes tarefas cotidianas, levando, muitas vezes, à perda precoce da autonomia. Ressaltam ainda que níveis adequados de força muscular e flexibilidade são fundamentais para bom funcionamento músculo-esquelético e contribuem para a preservação de músculos e articulações ao longo da vida. Para Fidelis, Patrizzi, Walsh (2013) a maioria das perdas funcionais se acentua com a atividade ineficaz do sistema neuromuscular, com o desuso e à diminuição do condicionamento físico, levando a complicações.

\section{CONCLUSÃO}

As atividades físicas realizadas no programa de orientação e reequilíbrio postural em grupo beneficiaram seus participantes em relação à mobilidade funcional e a flexibilidade além de contribuir para o equilíbrio dinâmico. Esses benefícios geram modificações positivas na qualidade de vida desses indivíduos, favorecendo o desempenho das atividades de vida diária e possibilitando movimentos sem restrições e sem dor, minimizando o risco de lesões.

\section{REFERÊNCIAS}

1. ALMEIDA, T. T.; JABUR, N. M. Mitos e verdades sobre flexibilidade: reflexões sobre o treinamento de flexibilidade na saúde dos seres humanos. Motricidade, v. 3, n. 1, p. 337-344, 2007. http://dx.doi.org/10.6063/motricidade.3(1).687

2. BERTOLLA, F. et al. Efeito de um programa de treinamento utilizando o método Pilates ${ }^{\circledR}$ na flexibilidade de atletas juvenis de futsal. Rev. Bras. Med. Esporte, v. 13, n. 4, Jul./Ago., 2007.

3. BADARO, A. F. V.; SILVA, A. H.; BECHE, D. Flexibilidade versus Alongamento: esclarecendo as diferenças. Revista Saúde, v. 33, n. 1, p. 32-36, 2007.

4. BANKOFF, A. D. P. et al. Postura e equilíbrio corporal: um estudo das relações existentes. Movimento e percepção, v. 6, n. 9, jul./dez., 2006.

5. CARNAVAL, P. E. Medidas e avaliação em ciências do esporte. $5^{a}$ ed. Editora Sprint, 2002.

6. FARIA, Jr., J. C.; BARROS, M. V. G. Flexibilidade e Aptidão Física Relacionada à Saúde. Revista Corporis, v. 3, n. 3, 1998.

7. FIDELIS, L. T.; PATRIZZI, L. J.; WALSH, I. A. P. Influência da prática de exercícios físicos sobre a flexibilidade, força muscular manual e mobilidade funcional em idosos. Rev. Bras. Geriatr. Gerontol., v. 16, n. 1, p. 109-116, Rio de Janeiro, 2013.

8. FIGUEIREDO, K. M. O. B.; LIMA K. C.; GUERRA R. O. Instrumentos de avaliação de equilíbrio corporal em idosos. Rev Bras Cineantropom Desempenho Hum., v. 9 p. 408-13, 2007. 
9. HASHIMOTO, B. et al. Análise da postura de participantes de um programa postural em grupo. Revista Eletrônica de Fisioterapia da FCT/UNESP, v. 1, n. 1, 2009.

10. MANTOVANI, F. A. et al. Análise do efeito de um programa de exercício sobre as medidas da cifose e lordose em pacientes com escoliose idiopática. Colloquium Vitae, v. 3, n. Especial, jul/dez., 2011.

11. MARTINS, M. R. I. et al. A eficácia da conduta do Grupo de Postura em pacientes com lombalgia crônica. Rev. Dor, v. 11, n. 2, p. 116-12, 2010.

12. MELLO, E. D.; LUFT, V. C.; MEYER, F. Atendimento ambulatorial individualizado versus programa de educação em grupo: qual oferece mais mudança de hábitos alimentares e de atividade física em crianças obesas? J. Pediatr., v. 80, n. 6, p. 468-474, 2004. http://dx.doi.org/10.1590/S0021-75572004000800008

13. NASCIMENTO, F. A.; VARESCHI, A. P.; ALFIERI, F.M. Prevalência de quedas, fatores associados e mobilidade funcional em idosos institucionalizados. Arquivos Catarinenses de Medicina, v. 37, n. 2, 2008.

14. PODSIADLO, D.; RICHARDSON, S. The Time "Up \& Go": A Test of Basic Functional Mobility for Frail Elderly Persons. Journal of the American Geriatrics Society, v. 39, n. 2, p. 142-148, 1991.

15. POLACHINI, L. O. Estudo comparativo entre três métodos de avaliação do encurtamento de musculatura posterior de coxa. Rev. Bras. Fisioter., v. 9, n. 2, p. 187-193, 2005.

16. RIBEIRO, C. C. A. et al. Nível de flexibilidade obtida pelo teste de sentar e alcançar a partir de estudo realizado na Grande São Paulo. Rev. Bras. Cineantropom. Desempenho Hum., v.12, n. 6, p. 415-421, 2010. http://dx.doi.org/10.5007/1980-0037.2010v12n6p415

17. SHUMWAY Cook A.; Brauer, S.; Woollacott, M. Predicting the Probability for Falls in CommunityDwelling Older Adults Using the Timed Up \& Go Test. Physical Therapy, v. 80, n. 9, p. 896-903, 2000. 